it is manifest that there are yet, in this State, many more lunatics, whose friends or guardians wish to place them in suitable establishments, and who would be restored or benefited by such procedure, than there are rooms for them in all our hospitals. Some of those who are thus rejected for want of room, are recent cases, and curable ; but for want of opportunity of recovery, are doomed to permanent insanity, and must remain, for life, burdens upon their estates or friends, or the public, for support. Others would be much more comfortably and easily maintained in proper hospitals, than they can be in any other places.

It therefore behooves a prudent, far-seeing and generous community to make such provision for lunatics, that none shall remain permanently diseased, if they can be restored; and if there are not yet means enough already provided for this purpose, to add more, until all the want shall be satisfied. And the facts presented to us show, that the want is not yet satisfied in this State.

\title{
COD.LIVER OIL.
}

BY D. M'kUR:R, M.1)., BANGOH, Mr.

[Comm nulcuted for the Bonton Medical und Surpical Journal.]

There have been few articles introduced into the materia medica with inore apparent reluctance, on the part of the profession, than the codliver oil ; but having once obtained a place in the catalogue of remedies, we predict it will be as slowly discarded as it has been adopted.

Cod-liver oil has been held as a popular remedy among the inhabitants of the sea coasts of Northern Europe for many centuries. The virtues imputed to it have been both vague and various, but it has been universally esteemed in many chronic diseases as a remedy of unequivocal power. Among the fishermen of our own Continent, it has long been highly valued for its curative power in rheumatism and its analogous diseases. It first attracted the attention of some medical practitioners in 1771 ; when Dr. Percival used it in many cases of chronic rheumatism, with success. Dr. Key, of Manchester, employed it extensively in hospital pracfice in 1789 ; and Dr. Bardsley used it previous to 1807. But it was not until 1822 that the attention of the profession was particularly directed to it by any publication based upon numerous and authentic cases in which it had been used. Dr. Schenks, of Germany, published in "Hufeland's Journal" of 1822-6, a series of papers in favor of its curative powers, which has led to its gradual adoption as a remedy in that country. More recently it has been employed in Belgium, France, England and Scotland. Witlin the last three years it hàs been introduced into the practice of medicine in this country, and from the numerous advertisements of those who prepare it for medical purposes, as well as from the reports that appear daily in the public journals, of its efficacy in disease, we infer that it is being used very generally.

Its therapeutic properties led Dr. Kopp; of Germany, to suspect that 
it contained iodine. This conjecture was verified by Drs. Loome, Bennett and others, who all, however, differ in regard to the quantity of iodine found in a given quantity of the oil, varying from 1-2 to 1-12 of one per cent. 'The proximate elements of the oil bear some resemblance to those of bile, as it contains in common with that fluid, oil, resin, and saline matter: It reddens litmus paper, is soluble in alcohol and ether, and is usually found to possess the specific gravity of 0.9:30. Ginelin obtained a saline mass from the oil which struck a deep-blue color with a solution of starch, and had all the usual re-actions of a solution of iodine.

In 18:35, Dr. Brefield published a monograph on the use of the codliver oil, in which he says, that from careful observation of many successful experiments with it in cases of rheumatism, he considers it a specific in both the acure and chronic forms of that disense. This testimony has since been corrolorated by a host of medical writers in Europe, who, likewise, agree that it merits the first place among anti-scrofulous remedies. Dr. Tanflied reports an instance of its success in curing a case of strumous caries of the spine, with extensive ulceration. Dr. Graves declares that he has seen it accomplish cures in scrofulous disease of the lymphatic glands, alier all other remerlies had been tried and failed. Drs. Richerand and M. Hall highly extol its curative powers in many obstinate cutaneous diseases; and other medical authorities, of equal respectability as the foregoing, have strongly recommended it in phthisis, chorea, spinal irritation, epilepsy, chlorosis, coxalyia, and tabes mesenterica.

The sensible therapeutic action of the oil seems to be almost null. Dr. Dunglison says. that it has no manifest effect on the secretions, [excretions?], except occasionally on the urinary and cutaneous depurations; on the healthy organization it seems to excite no marked change. In strumous affections, however, its favorable influence is striking, as well as in rheurmatic and gouly disorders. In 71 patients for whom Dr. Bennett prescribed the oil, he observed that vomiting was occasioned by it in three cases; slight diarrhna in seventeen cases; slight diuresis in two cases; and diaphoresis in twelve cases. He thinks it owes its efficacy in scrofula to its iodine, and attributes its superiority over that obtained by chenical means to its organic combination with animal matter.

Dr. Acherson, of Berlin, explains its morlus operandi in another manner. He conceives that oil globules are essential to the formation of the elementary cells of tissue ; for the elementary globule is composed of liquid fat and albumen ; and observes, that when fat in a liquid state and albumen are brought into contact, a small quantity of the latter forms a case around a rlobule of the former, in the same manner as the formative rudiments of animal tissue are produced fiom a combination of the oil contained in bile and the albumen of the chyle.

Dr. Bennett and most other writers, while acknowledging the ingenuity of Acherson's hypothesis, differ from him, by attributing its curative power to its agency in improving the function of nutrition, thereby affording to the blood an energetic and rich plasmi, and promoting the activity of the vital forces, and forwarding the absorption of morbid deposits. In addition to these explanations, Dr. C. J. B. Willians 
thinks, that " its peculiar fuidity and little proneness to change enables it to pervade all structures and to penetrate even into imperfectly organized deposits, and by softening their concrete fatty molecules, and rendering more permeable and supple their whole mass, brings them more under the influence of the adjoining living parts, through the circulation; in which either their vitality and nutrition are improved and maintained, or if incapable of improvement, they are gradually dissolved and absorbed away."

These opinions all tend to establish its character as a nutritive of no ordinary power. In regard to its formative or solvent agencies, all is conjecture; its claims to these properties seem to have been advanced to explain its wonderful eflects in cachectic diathesis, and scrofinlous deposits. But when we consider that both of these morbid conditions are but the effect of vitiated or imperfect assimilation, the ingenious theories of Acherson, as well as Williams, will be found to be wholly gratuitons.

In addition to the several explanations, already advanced, of its modus operandi, we feel authorized, from having olsserved its eflects in some peculiar diseases, to attribute its restorative powers to its direct adnissibility into the circulation, without undergoing the process of digestion, or passing through the lacteal system. We do not suppose that all the oil that enters the stomach, is in this manner disposed of, or that none goes the usual route of the other digestible matter; but we think we have strong reasons for the opinion, that much of it finds its way in a more direct manner into the circulation, either by vital absorption or by endosmosis. Its low specific gravity would seem to favor its transmission by the latter method. However, the following case is one of those alluded to, which have led us to the foregoing opinion.

J. M. C., a young girl, aged 4 years, was attacked with cholera infantum during the autumn of 1848 , which resulted in a diseased condition of the mesenteric glands; thejr enlarged and indurnted state could be distinctly felt through the walls of the abdomen. The emaciation peculiar to that disease steadily progressed, in defiance of every reniedy, as well as the gratification of a voracious appetite; until the case became so hopeless that all medication was suspended, except such dietetic regulations as.were found to hest insure the temporary comfort of the patient. The case, under this expectant treatment, for weeks continued to advance towards an apparent fatal termination; when, about a month ago, in consequence of the earnest solicitation of the mother, that something more might be attempted to save her child, the cod-liver oil was directed to be given, in half-tablespoonful doses three times a-day. About a week after the commencement of the oil, an evident improvement in the general appenrance of the child was perceptible; although the discharges from the bowels remained as chylous, and as frequent as they were before the oil was given. This improvement is still going on. The pulse has nearly doubled its volume; and the glands, which were formerly easily discernible by the hand, cannot now be readily felt, in consequence of the increased thickness of the walls of the abdomen. Whether this patient will recover or not, is still doubtful; but the change in the increase of adipose tissues, as well as the improvement of the general circulation, since the commencement of the oil treaiment, irresis- 
tibly leads to the inference, that nutritive elements have found a way into the circulation different from the usual route of digestion. The declarations of other patients that the oil acts as a cordial, as promptly and as effectually as an equal quantity of good wine, strengthens this supposition.

'The curative powers of the cod-liver oil are more manifest in tubercular phthisis than in any other disease in which we have prescribed it. Indeed, no therapeutic agent has come under our observation, which has apparently produced such favorable changes in this disease, as the oil. Undoubtedly much of its efficacy depends upon its power of invigorating the system, and increasing the "turgor vitalis." Yet we apprehend it sometimes acts in a conservative capacity, by presenting to the oxygen that enters the circulation a supply of hydro-carbonaceous elements, to satisfy its imperative alfinities; and in this manner it tends to protect the nitrogenous tissues from rapid decomposition. This hypothesis receives some support fiom the results of a chemical and microscopical examination of three specimens of urine, from each of three individuals, both before and after taking the oil; when the urea was found in much less quantity in the urine passed after taking the oil, than in the specimens obtained before its use.

Within the last two years we have prescribed the cod-liver oil in thirteen cases of well-marked tubercular phthisis, and the following have been the results. It has been discontinued in two cases; in consequence of its producing hemoptysis in one case, and diarrhca in the other. Two cases have not perceived any effect whatever from it. Five cases have been materially benefited; three of whom are yet persisting in its use. And four cases are to all appearances cured; they have regained their flesh and strength, are free from cough or hurried respiration, and their circulations have returned within the limits of health.

In one case of caries of the spine, of a strumous character, when the patient was unable to sustain the upright position without great suffering at the time when the oil was first taken, about four months ago, it has so improved his condition that he is able to take part with other children in their pastimes out of doors, with perfect freedom from pain.

Although the effect of cod-liver oil on a healthy system is almost null, yet it ought not to be inferred that no discrimination is necessary in its administration in disease ; for there are some pathological states in which it proves highly injurious. Diseases attended with intense arterial action, increase of heat, intestinal irritation, or hemorrhagic tendencies, are those morbid conditions with which I have found it to be incompatible.

If this communication will induce some of the readers of the Journal to give the cod-liver oil a more extensive trial, and report their experience of its efficacy in disease, my object will be accomplished.

March 7, 1849. 